\title{
QUANTIFICATION OF DIRECTION AND MAGNITUDE OF CYCLICAL POSTURAL SWAY USING ELLIPSES
}

\author{
Patrick J. SParto ${ }^{1,2}$, Mark S. Redfern ${ }^{1,2.3}$ \\ 'Department of Physical Therapy, ${ }^{2}$ Department of Otolaryngology \\ ${ }^{3}$ Department of Bioengineering. University of Pittsburgh, Pittsburgh, PA
}

\begin{abstract}
In this paper we demonstrate a new method to quantify direction and magnitude of sway in response to periodic inputs. The postural sway response was modeled as an ellipse, allowing the determination of angle of heading as well as the resultant magnitude. To demonstrate this methodology, center of pressure data obtained from a subject receiving sinusoidal $(0.25 \mathrm{~Hz}, 1.2 \mathrm{~mA}$ peak-to-peak) galvanic vestibular stimulation in both the binaural-bipolar and binaural-monopolar configurations were analyzed. The binaural-bipolar and binaural-monopolar stimuli elicited sway patterns that were oriented at $4^{\circ}$ and $97^{\circ}$ to the medial-lateral axis, respectively. In addition, the binaural-monopolar stimulus generated twice as much sway as the binaural-bipolar stimulus. We propose that this method can be applied to sway obtained from sinusoidal inputs to the sensory systems controlling balance. Estimation of the direction and magnitude of postural sway will become an important tool for understanding postural control mechanisms for disturbances to balance that do not occur in a cardinal direction.
\end{abstract}

Biomed Eng Appl Basis Comm, 2001 (October); 13: 213-217.

Keywords: balance, galvanic vestibular stimulation, vision, somatosensory, posture

\section{INTRODUCTION}

Analysis of postural sway pattems has been used frequently to characterize the relative health of the postural control system.(Baloh et al., 1998; Maki ct al., 19\%) Most often, inferences are made based on some measurc of the magnitude of the movement, such as: root-mean square position. peak-to-peak position, area of excursion and average sway velocity.(Maki ct al., 1990; Prieto el al., 1996) Rarely is the direction of sway considered, primarily because the perturbations

Received: Ocl. 2, 2001; accepted: Oct. 17, 2001

Correspondence: Patrick Sparlo, PhD, PT

Universily of Pittsburgh

Department of Physical Therapy

6035 Forbes Tower

Pittsburgh, PA 15260, USA

Email: psparto@pitt.edu to the sensory systems serving balance are oriented alung the anterior-posterior (A-P) and medial-lateral (M-L) axes. In order to increase our understanding of postural control, we believe that it is necessary to extund these experiments to include perturbations that are not along the primary axes. Although in many cases there is a dominam cardinal direction of sway, reflecting stabilizing actions occurring at the ankles (A-P) and hips (M-L),(Winter et al., 1996) a unidirectional analysis may mask important interactions in these postural control strategits. Consequently, it may he helpful to determine if there is a preferential direction of sway that lies berween these two orthogonal directions.

Often, the contributions of the various sensory modalities (e.g. vestibular, visual, and somatosensory) to postural control are studied using sinusoidal inputs.(Cuats, 1972; Diener el al., 1982; Peterka and Benolken, 1995) For example, consides the stabilogram displayed in Figure 1, which was generated by a subject receiving sinusoidal galvanic electrical vestibular stimulation. It is clear that the anterior-posterior (A-P) component of sway is grcater than the medial-lateral 
(M-L) component. However, the M-L component is not insignificant. A quantification of the direction of the response would be useful in determining the sensorimotor response to the stimulus.

We propose that fitting postural sway data with an ellipse can conveniently parameterize the magnitude and direction of the postural response obtained during cyclical sensory input. Importantly, the direction of the ellipse conveniently expresses information about the relative magnitude of A-P and $M-L$ sway components, thus reflecting the bivariate nature of the data. In addition, the magnitude of the major axis reflects the overall amount of sway.

\section{METHODS}

\subsection{Ellipse Geometry}

As shown in Figure 2, an ellipse centered at the origin $(\mathrm{O})$ is defined by three parameters, the major axis (a), minor axis (b), and angle between the positive $x$ axis and the major axis $(\theta)$. The parametric equations defining this ellipse (Batschelet, 1981, p. 250), setting $\phi$ from $0^{\circ}$ to $360^{\circ}$, are:

$$
\begin{aligned}
& x=a * \cos \theta * \cos \phi-b * \sin \theta * \sin \phi \\
& y=a * \sin \theta * \cos \phi+b * \cos \theta * \sin \phi
\end{aligned}
$$

The implicit equation of the ellipse (Batschelet, 1981 , p. 251), which eliminates the variable angle $\phi$, is:

$$
A * x^{2}+2 * B * x * y+C * y^{2}=1
$$

where

$$
\begin{aligned}
& A=\frac{\cos ^{2} \theta}{a^{2}}+\frac{\sin ^{2} \theta}{b^{2}} \\
& B=\cos \theta * \sin \theta *\left(\frac{1}{a^{2}}-\frac{1}{b^{2}}\right) \\
& C=\frac{\sin ^{2} \theta}{a^{2}}+\frac{\cos ^{2} \theta}{b^{2}}
\end{aligned}
$$

Using equation 3, (A, B, C) can be computed in a least squares sense using multiple linear regression. Then $(a, b, \theta)$ are determined using equations 4-6. Since equations 4-6 are nonlinear, the following computational formulae can be used after eliminating intermediate steps (Batschelet, 1981, p. 256-257):

$$
\begin{aligned}
& a=\sqrt{\frac{2}{A+C-R}} \\
& b=\sqrt{\frac{2}{A+C+R}}
\end{aligned}
$$

$$
\theta=\tan ^{-1}\left(\frac{2 B}{A-C-R}\right)
$$

where

$$
R=\sqrt{(A-C)^{2}+4 B^{2}}
$$

Once $(\mathbf{a}, \mathrm{b}, \theta)$ are known, an ellipse fit to the postural sway data can be constructed by substituting these values into Equations 1-2 and setting $\phi$ to values between $0^{\circ}$ and $360^{\circ}$.

\subsection{Experimental Procedure}

Center of pressure (COP) data were obtained from a healthy subject standing with feet together while receiving sinusoidal galvanic vestibular stimulation (GVS). A peak-to-peak $1.2 \mathrm{~mA}, 0.25 \mathrm{~Hz}$ GVS was delivered for $\mathbf{4 0}$ seconds in both binaural-monopolar and binaural-bipolar configurations, resulting in $10 \mathrm{cy}-$ cles of stimulation. Three trials of each stimulus were conducted. The COP data were obtained from a 6 degree of freedom force platform (Bertec, Inc.) at a sampling frequency of $100 \mathrm{~Hz}$. Although the COP data contains power in the frequency range $0-2 \mathrm{~Hz}$, only the power that resides at the stimulus frequency is of interest in this analysis. In order to isolate this response at $0.25 \mathrm{~Hz}$, the data was bandpass filtered in the range $0.20-0.30 \mathrm{~Hz}$ (Butterworth, $2^{\text {nd }}$ order, 0 phase lag). The raw and the filtered signals are displayed in Figure 3 for the stabilogram shown in Figure 1.

\subsection{Data Analysis}

After discarding the first and last cycles, the filtered A-P and M-L COP data were divided into 8 cycles based on the endpoints of each cycle of GVS. The

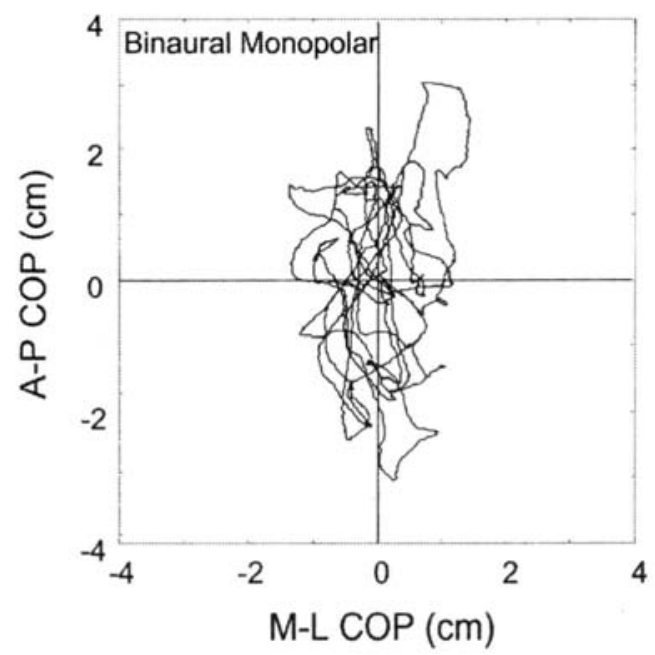

Fig. 1 Raw stabilogram of center of pressure (COP) data obtained during application of binauralmonopolar (BM) galvanic vestibular stimulation. 


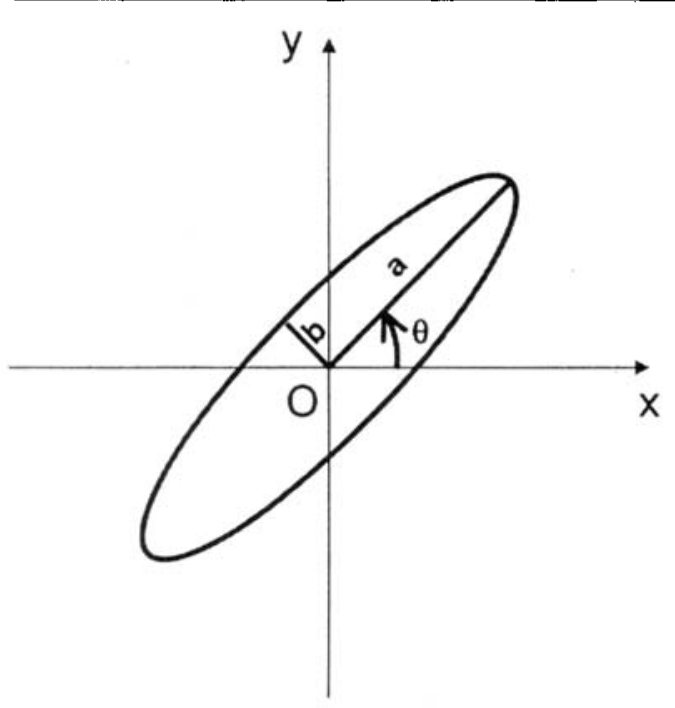

Fig. 2 Definition of parameters of ellipse centered at the origin $(0)$. $x$ : medial-lateral component of center of pressure. $y$ : anterior-posterior component of center of pressure. $\theta$ : direction of ellipse with respect to the medial-lateral axis. a: major axis length from origin. $b$ : minor axis length from origin.

8 cycles of data were ensemble-averaged to reduce the considerable cycle-by-cycle variation in shape and magnitude of stabilogram. The A-P and M-L data were then subjected to the elliptical fitting routine. Mean values of the direction, and major and minor axis lengths from the three trials were computed using directional statistics and linear statistics, respectively. (Batschelet, 1981; Mardia and Jupp, 2000; Neter et al., 1996)

\section{RESULTS}

The bandpass-filtered, ensemble-averaged stabilograms and least-squares ellipses obtained during a single trial of binaural-monopolar (BM) and binauralbipolar (BB) stimuli are displayed in Figure 4. Clearly, the ellipses fit the processed COP data well. The parameters of the ellipse $(a, b, \theta)$ were computed for the COP profiles generated by both stimuli. The computed values show that the direction and major axis length of the COP for the two stimuli are distinctly different

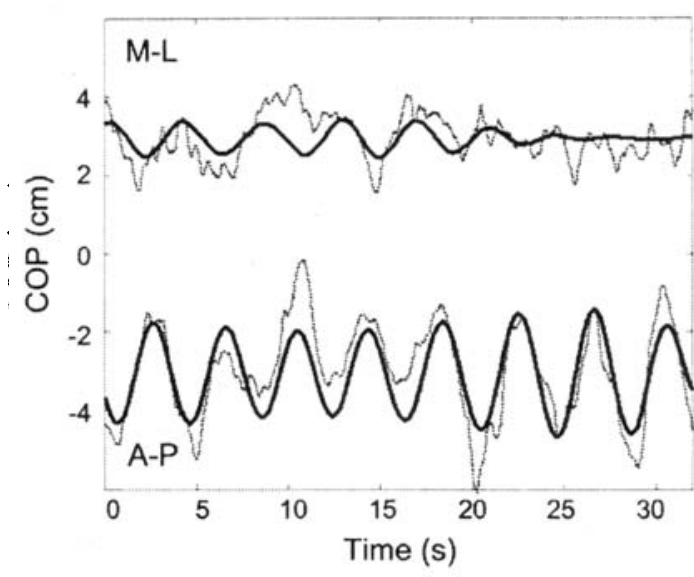

Fig. 3 Raw (dotted line) and $0.25 \mathrm{~Hz}$ bandpass filtered (solid line) components of center of pressure data that are displayed in Fig. 1.

(Table 1). The direction of the ellipses obtained from the binaural-monpolar stimulus was close to the anterior-posterior axis $\left(97^{\circ}\right)$. Meanwhile, the binauralbipolar stimulus elicited movement close to the anterior-posterior axis $\left(4^{\circ}\right)$. In addition, the binauralmonopolar stimulus generated approximately twice the response as the binaural-bipolar stimulus.

\section{DISCUSSION}

The technique proposed in this paper presents several advantages over previous methods of analyzing postural sway data where direction may be important. For one, sway is not considered to occur independently in both the A-P and M-L directions. Rather, the sway is considered to be a bivariate distribution. Granat et al. (Granat et al., 1991) proposed another method for quantifying the direction of postural sway that depended on minimizing the distance of the twodimensional postural sway data points from a line through the centroid of the data. One drawback to using this iterative method is that the data points are considered to be independent, when in fact the points are spatially and temporally correlated. Alternatively, our analysis considers the data points to be a continuous time series. Another benefit is that both magnitude and direction of sway are computed simultaneously in a

Table 1. Computed mean (s.d.) ellipse parameters for the two sinusoidal galvanic vestibular stimulation conditions.

\begin{tabular}{c|c|c|c}
\hline Stimulus & Angle $(\theta)$ & Major axis (a) & Minor axis (b) \\
\hline Binaural Monopolar & $97(7)^{\circ}$ & $2.0(0.6) \mathrm{cm}$ & $0.06(0.03) \mathrm{cm}$ \\
Binaural Bipolar & $4(17)^{\circ}$ & $1.0(0.1) \mathrm{cm}$ & $0.6(0.1) \mathrm{cm}$ \\
\hline
\end{tabular}



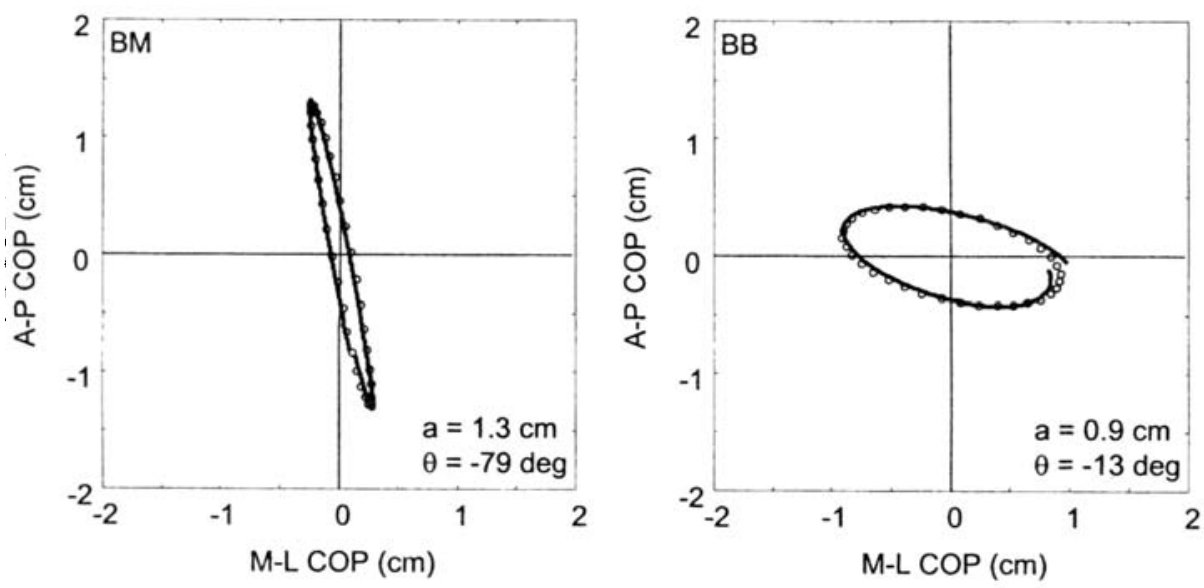

Fig. 4. Elliptical fit (open circles) to processed stabilograms (solid lines) for center of pressure data obtained during binaural-monopolar (BM, left) and binaural-bipolar (BB, right) galvanic vestibular stimulation. Direction $(\theta)$, and major axis (a) parameters labeled on each plot. The elliptical fit for the binauralmonopolar stimulus was calculated from the data shown in Figures 1 and 3.

least-squares sense. Hence, information about the magnitude of sway is preserved.

The results demonstrated that the mean angle was 4 degrees for the binaural-bipolar stimulus trials and 97 degrees for the binaural-monopolar. These values correspond well to the observations that the magnitude of sway obtained during galvanic vestibular stimulation is greater in the medial-lateral direction for a binaural-bipolar stimulus and greater in the anteriorposterior direction for a binaural-monopolar stimulus. (Day et al., 1997; Magnusson et al., 1990; Pavlik et al., 1999; Severac Cauquil et al., 1998) However, our analysis takes another step by specifying direction. Understanding the direction of sway may reveal the strategies utilized by the postural stabilizing muscles of the ankle and hip. In addition, the binauralmonopolar stimulus elicited about twice as much sway response as the binaural-bipolar stimulus. This finding may be attributed to the greater functional base of support in the A-P direction compared with the M-L direction while standing in the feet together position.

In this paper, a methodology was presented that quantifies the direction and magnitude of postural sway data obtained during sinusoidal galvanic vestibular stimulation. In addition, we believe that this technique would also work well with postural sway data that results from visual or somatosensory perturbations. This methodology may help to elucidate the postural control mechanisms that occur as investigations consider challenges to balance that do not occur in a cardinal direction.

\section{REFERENCES}

1. Baloh, R. W., Jacobson, K. M., Beykirch, K., Honrubia, V., 1998. Static and dynamic posturography in patients with vestibular and cerebellar lesions. Archives of Neurology 55, 649-654.

2. Batschelet, E., 1981. Circular Statistics in Biology. Academic Press, London.

3. Coats, A. C., 1972. The sinusoidal galvanic bodysway response. Acta Oto-Laryngologica 74,155 162.

4. Day, B. L., Severac Cauquil, A., Bartolomei, L., Pastor, M. A., Lyon, I. N., 1997. Human bodysegment tilts induced by galvanic stimulation: a vestibularly driven balance protection mechanism. Journal of Physiology 500, 661-672.

5. Diener, H. C., Dichgans, J., Bruzek, W.,Selinka, H., 1982. Stabilization of human posture during induced oscillations of the body. Experimental Brain Research 45, 126-132.

6. Granat, M. H., Barnett, R. W., Kirkwood, C. A., Andrews, B. J., 1991. Technique for calculating the direction of postural sway. Medical \& Biological Engineering \& Computing 29, 599-601.

7. Magnusson, M., Johansson, R.,Wiklund, J., 1990. Galvanically induced body sway in the anteriorposterior plane. Acta Oto-Laryngologica 110, 1117.

8. Maki, B. E., Holliday, P. J.,Fernie, G. R., 1990. Aging and postural control. A comparison of spontaneous- and induced-sway balance tests. Journal of the American Geriatrics Society 38, 1-9.

9. Mardia, K. V., Jupp, P. E., 2000. Directional Statistics. John Wiley and Sons, Chichester, England. 
10. Neter, J., Kutner, M. H., Nachtsheim, C. J., Wasserman, W., 1996. Applied Linear Statistical Models. McGraw-Hill, Boston.

11. Pavlik, A. E., Inglis, J. T., Lauk, M., Oddsson, L., Collins, J. J., 1999. The effects of stochastic galvanic vestibular stimulation on human postural sway. Experimental Brain Research 124, 273-280.

12. Peterka, R. J.,Benolken, M. S., 1995. Role of somatosensory and vestibular cues in attenuating visually induced human postural sway. Experimental Brain Research 105, 101-110.

13. Prieto, T. E., Myklebust, J.B., Hoffmann, R.G.,
Lovett, E.G., Myklebust, B.M., 1996. Measures of postural steadiness: Differences between healthy young and elderly adults. IEEE Transactions on Biomedical Engineering 43, 956-966.

14.Severac Cauquil, A., Gervet, M. F., Ouaknine, M., 1998. Body response to binaural monopolar galvanic vestibular stimulation in humans. Neuroscience Letters 245, 37-40.

15. Winter, D. A., Prince, F., Frank, J. S., Powell, C., Zabjek, K. F., 1996. Unified theory regarding A/P and $\mathrm{M} / \mathrm{L}$ balance in quiet stance. Journal of Neurophysiology 75, 2334-2343. 